\title{
The Safe Navigation of Partial Motion Planning Based on "Cooperation" with Roadside Fixed Sensors in VANET
}

\author{
Rong Ding ${ }^{1,2}$, Xiaoguang $\mathrm{Li}^{1,2}$ \\ ${ }^{1}$ State Key Laboratory of Software Development Environment, Beihang University, \\ ${ }^{2}$ School of Computer Science and Engineering, Beihang University \\ Beijing, China \\ E-mail:dingr@buaa.edu.cn,lixiaoguang@cse.buaa.edu.cn \\ Received July 3, 2010; revised August 5, 2010; accepted September 7, 2010
}

\begin{abstract}
In recent years, many methods of safe vehicle navigation and partial motion planning (PMP) have been proposed in vehicular ad-hoc network (VANET) field. In order to improve the limitation of traditional PMP, this paper presents a novel effective way to plan motion with cooperation of roadside fixed sensors (RFSs). With their cooperation, the vehicles can get the surrounding information quickly and effectively, and give highly accurate projections about the near future conditions on road. After proposing our algorithm, the worst case is analyzed and methods are found to solve the problem. Finally we conduct one elemental contrast experiment, driver situation awareness, with or without the "cooperation" of RFSs in highway scenarios. The result shows that the vehicles can make a better PMP based on the forward conditions received from RFSs, and extend the warning distance obviously when emergency happens.
\end{abstract}

Keywords: Partial Motion Planning, Safe Vehicle Navigation, Roadside Fixed Sensors, Cooperation

\section{Introduction}

It is a dream of human beings to achieve autonomous mobile vehicles since the invention of cars. With the rapid development of science and technology, and through the persistent and unremitting efforts by scientists, many novel tools which are equipped with modern technologies are used in cars such as GPS navigators and PSD sensors. By using those tools, unnecessary accidents can be avoided and destination can be achieved easily as well [1].

However, there is still a long way to make the dream come true. The tools are only used to human in order to make the driving safer, but it cannot take the place of human's driving so far. The 2007 DARPA Urban Challenge presented the fact that there are plenty of works to do if achieving fully autonomous driving especially in urban environment with the extremely complicated conditions, such as traffic lights and pedestrians, and safe navigation is also a huge problem.

In safe navigation field, reaching a goal and avoiding collisions are two main purposes and the analysis is carried out in detail [2]. So far, a proven method is the layer structure. In that structure, the safe navigation architec- ture can be divided into five layers as follows, mission manager, route planning, partial motion planning (PMP), low level components, and hardware layer [3]. Nowadays, with the GPS's development, the research emphasis has been changed to partial motion planning.

There are two main paradigms in partial motion planning [4]. They are the plans based on prior information and real time information. And the latter seems to be safer and more reliable. Several schemes have been presented firstly in mobile robots $[5,6]$. In real time model, the systems are obliged to make a relatively correct decision within a restricted time. If not, the vehicles may be in danger passively.

Nowadays, in vehicular ad-hoc networks (VANETs), cars can be equipped with communication device so as to exchange information about position, speed, traffic jams or other road conditions. Safe navigation is one of the VANETs applications. Vehicle to vehicle communications provide a new way of collision warning forwarding and intersection collision warning.

This paper is organized as follows: Section 2 briefly reviews the previous work and the limitation that is not resolved completely. And in Section 3, an effective improvement is provided: the "cooperation" with RFSs, 
with details of the algorithms to achieve "cooperation" in Section 4. Then contrast experiments are carried out in Section 5 and Section 6 concludes the paper.

\section{Previous Work and the Limitation}

\subsection{Previous Work}

In this section, we discuss the related work on safe navigation of Partial Motion Planning.

On hazardous terrain, a fuzzy logic approach is advanced in [7]. In that paper, navigation strategy is comprised of three simple and independent behaviors: seek-goal, traverse-terrain and avoid-obstacle. Those three behaviors are combined through appropriate weighting factors in order to generate the final steering and speed commands. The weighting factors are produced by fuzzy logical rules that are taken the current status into account. Also in [8], they employ fuzzy reasoning to allow for aggregation decisions based on a flexible and extensible set of criteria. These criteria can be application specific and enable a dynamic fragmentation of the road according to the applications' requirements.

In order to acquire safe motion planning, Inevitable Collision State (ICS) has been characterized by [9]. The most important contribution of ICS is that it promotes a dynamic relationship properly between collision states and safety states. Thus we can guarantee stronger safety and giving a safe partial motion planning.

In [4], ICS takes into account the dynamics of both the system and the moving obstacles. The core of safety issues is computing ICS-free partial motion in the shortest period of time. The paper [4] presents a property in order to reduce the complexity of the PMP algorithm, which simplifies the safety checking of a trajectory. If one partial trajectory is an ICS-free partial motion, the trajectory is collision free.

Another safe navigation technology is using virtual stick [10]. They propose a reliable method for navigating the robot to the target point, and draw an environment map using infrared sensors. However, it is difficult to distinguish distance information using only one-dimensional image information. So they use a virtual stick that represents different distances with different colors to improve the perception of the position and distance of the obstacle.

\subsection{Limitation}

All above methods only concern the one-car situation which is myopia. We cannot get enough information of the driving environment only by sensors or measure in- frastructure equipped on a single car, especially in the complex and volatile urban scenarios. Each car needs to be kept in contact with others nearby. However, the number of cars is huge and they are all in motion. Those external factors have made it more difficult to ensure the reliable connections.

One significant reason of "partial" lies in the real-time limitation in PMP. In an environment featuring moving objects, you have to decide upon the further action within limited time, otherwise you would take the risk of being hit by another moving object. Getting the conditions of front road sections in time can short the PMP processing time.

In addition, a prior information updater does not exist. Even if there was one, the prior information would not update in time. Prior information would be collected by single car with the whole system in a lack of sink, as a result, cars would fail to send what they have already collected and therefore could not acquire front road condition.

\section{An Effective Improved Method: the "Cooperation" with RFSs}

In this part, we propose a novel approach to solve problems mentioned above. We introduce "cooperation" with RFSs into PMP. Figure 1 shows the structure of "cooperation". Cars send and receive messages from RFSs, and RFSs can transmit these messages to each other. The latest technology is applied to enable every single car to detect its own possible collision, but ignore the transmission among cars. In a word, messages are transmitted from car to RFS, from RFS to car, and RFS to RFS. In Section 4 , the three types of messages will be expounded in detail.

Traditional safe navigation of partial motion planning is based on single cars. In their opinion, if one car manages to do well in collision avoidance, the whole work would be finished. However, that is very shortsighted. One successful navigation even one successful partial motion planning depends on the accuracy grade of real-time information and front-road condition. In this case, RFSs are used as sink, and they can aggregate messages from cars and transmit warning messages or other significant messages from each other. In this way, as is shown in Figure 1, for example, Car5 can hardly get known about the condition of Car1 by itself when Car1 is collided, because Car3 has covered Car5's sight. But collision messages can be transmitted from RFS1 to RFS2, and then to RFS3, and at the same time both RFS2 and RFS3 send warning messages to cars nearby. In this way, Car5 manage to acquire the front condition. 


\section{Algorithms to Achieve "Cooperation"}

In this section, we give an intimate design of three types of messages mentioned in Section 3, then put forward our novel algorithms in dynamic road scenarios, and at the end advantages of this cooperative methods will be summarized.

\subsection{Design of Three Types of Messages}

As is shown in Section 3, the three types of messages are from car to RFS (C-R), from RFS to car (R-C), and RFS to RFS (R-R). The basic message formats are shown in Figure 2.

As is shown in Figure 2, there are three types in this paper, 0 means C-R, 1 means R-C, and 2 means R-R. Len means the length of data segment. CRC is cyclic redundancy check, and it is used to check whether data transmission is true or not.

$\mathrm{C}-\mathrm{R}$ messages are the source knowledge of the system. Cars can get information from sensors which are attached to them. In this paper, attention is paid to the danger cars may meet. Each C-R message's source address is car's own ID, when moving into a new road section, cars will receive RFS's broadcast message. Immediately after cars get RFS's ID in source address of RFS's broadcast message, then cars begin to send C-R messages. Data segment of C-R messages contains the following information: car's velocity, acceleration, distance from forward obstacle, and some flag bit for RFS's judging safety, such as danger flag, ICS flag and emergency flag.

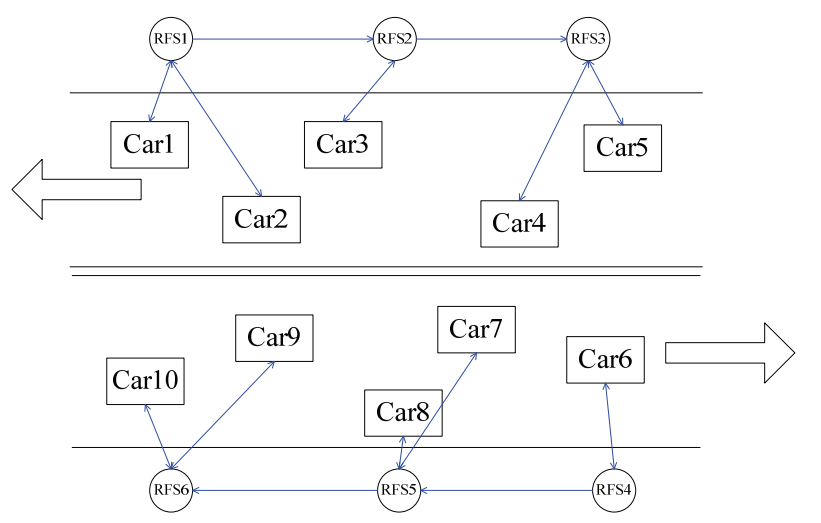

Figure 1. The structure of "cooperation" with RFSs.

\begin{tabular}{|c|c|c|c|}
\hline SRC ADDRESS & DST ADDRESS & Type & \multicolumn{2}{|c|}{ Len } \\
\hline \multicolumn{2}{|c|}{ Data } & CRC \\
\hline
\end{tabular}

Figure 2. Basic message format.
$\mathrm{R}-\mathrm{C}$ messages are always regarded as prior information, as RFSs sends warning alarm or motion suggestions based on conditions of front section. Therefore, source address of R-C is RFS's own ID, messages are sending in flooding way thus any cars which is in this section can get R-C messages. Therefore, destination address should be defined as "1" sequence. Data segment of R-C messages consist of following information: forward section cars (if in the middle of the road), forward intersection queue length (if in urban scenarios), forward highway exit queue length (if in highway scenarios). The most important data is flags of warning alarm and motion proposal. These flags are concerned of safety of drivers and passengers' lives and property.

$\mathrm{R}-\mathrm{R}$ messages are the information exchanged between each RFS on roadside, using cable transmissions in order to make sure the reliability while transmitting. Both source address and destination address are RFS's ID. We specially point out that source address is RFS's own ID, and destination address is upriver RFS's ID. That is because in traffic flow, if one car has passed the scene of accident, that car has no relationship to this accident. Data segment of R-R messages consist of following information: emergency state and RFS's ID of accident site (if has), numbers of passing cars in last 10s.

Based on the elementary design of message formats above, the algorithm of "cooperation" in dynamic scenarios will be discussed next.

\subsection{Algorithm of "Cooperation"}

"Cooperation" can be used similarly between highway scenarios and urban scenarios, although the two scenarios are very different.

In highway scenarios, all the obstacles are cars, thus the participators in the whole system are simpler and it is easier to monitor data and administer them. However, we cannot ignore the accidents happening on the highway. The velocity of each car reaches 120 kilometers per hour, that is to say, an ordinary car may run 10 meters or more in the time of a blink of an eye. Without a reliable warning system, it is very dangerous for those following cars while one car may suddenly have an accident. While in urban scenarios, obstacles can be cars, motorcycles, bicycles and pedestrians. Thus system must be very complex and it is more difficult to monitor a very exact real-time data. However, we should supply a reliable service to decrease the number of accidents, and cooperation with RFSs can provide all the cars around front road conditions and cars can analyze messages they have received and control their speed itself, and once they meet an accident forward they can take measures immediately. 
Figures 3(a) (b) (c) shows the brief flowcharts of algorithm for the RFSs at the intersections or highway exit, on the middle of the road and for the cars respectively.

As is shown in Figure 3(a), there is no prior RFS for the sensors who are settled at the intersections or highway exit. So they can only send interaction or exit messages to cars nearby in flooding way, thus they can receiving $\mathrm{C}-\mathrm{R}$ messages as feedbacks. Then analyze the received message and judge whether there exists an emergency flag. If so, then send emergency messages to the cars nearby and send danger message to the next RFS; if not, just record this C-R message in the buffer and regularly $(10 \mathrm{~s}$, e.g.) send road condition messages to next RFS based on the information recorded last period of time.

In Figure 3(b), we can observe that there are more assignments for those RFSs in the middle of roads. First they have to listen to receive messages so as to get information from prior RFS and once they have received R-R messages they can make a judgment whether danger flag is included. If so, they send warning message to cars nearby and send danger messages to next RFS in addition if original danger message is from prior RFS to make sure danger messages can send at least three RFSs to extend warning area. Their other assignments such as receiving $\mathrm{R}-\mathrm{R}$ messages exclude danger flag; receiving $\mathrm{C}-\mathrm{R}$ messages and dealing with $\mathrm{C}-\mathrm{R}$ messages are same as RFSs at the intersections or highway exit.

At last, we analyze Figure 3(c), which is cars' behavior. When drivers are driving into a new road section, what they can do is driving steadily and safely. But they have no idea of front road condition; as a result, they have to receive information passively. In our algorithm, just like a seasoned driver, cars receive messages from RFS around, and when cars get R-C message, analyze it and record RFS's ID so as to make contact with the nearest RFS, then check whether there exists emergency flag, if so, brake the car immediately, and if just exists warning flag, that means in the front section of the road, there happens an accident, so decelerate the car immediately, and else just control car's velocity as the case may be. It is worth notice that once the car meets an accident, an emergency brake should be carried out by the automatic control immediately, send collision message at the same time. Thus we can make sure the RFS can get road condition and notice other cars in time, and then the car have to wait for aids.

\subsection{Worst Case}

We must realize what the worst case is, and we should make measures to overcome the trouble. In this system, we enumerate three bad cases as follows, the failure of
RFS, the failure of Cars and substantial cars on one section. In this part, we discuss the method to solve them.

Once the RFS lost efficacy and the supervision on the road section may be missed. While one car runs into a new section, and RFS of that section is right out of work, it cannot get $\mathrm{R}-\mathrm{C}$ messages and once it gets a traffic accident, it cannot be helped to send emergency messages and as a result, "cooperation" does not work and cars behind the traffic accident scene will not receive any warning message.

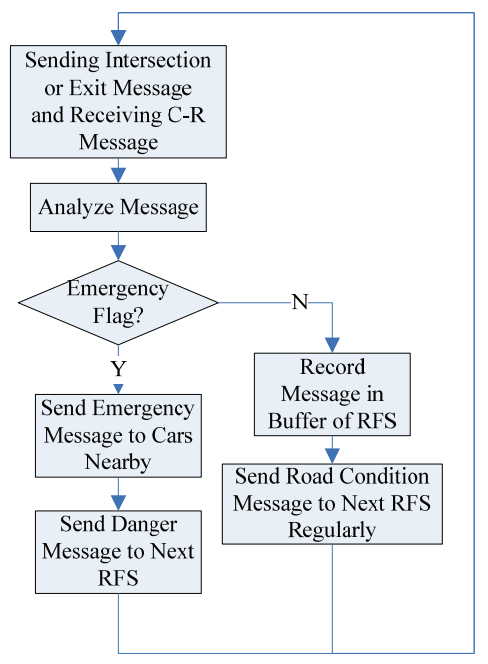

(a) Brief flowchart for the RFSs at the intersections or highway exit.

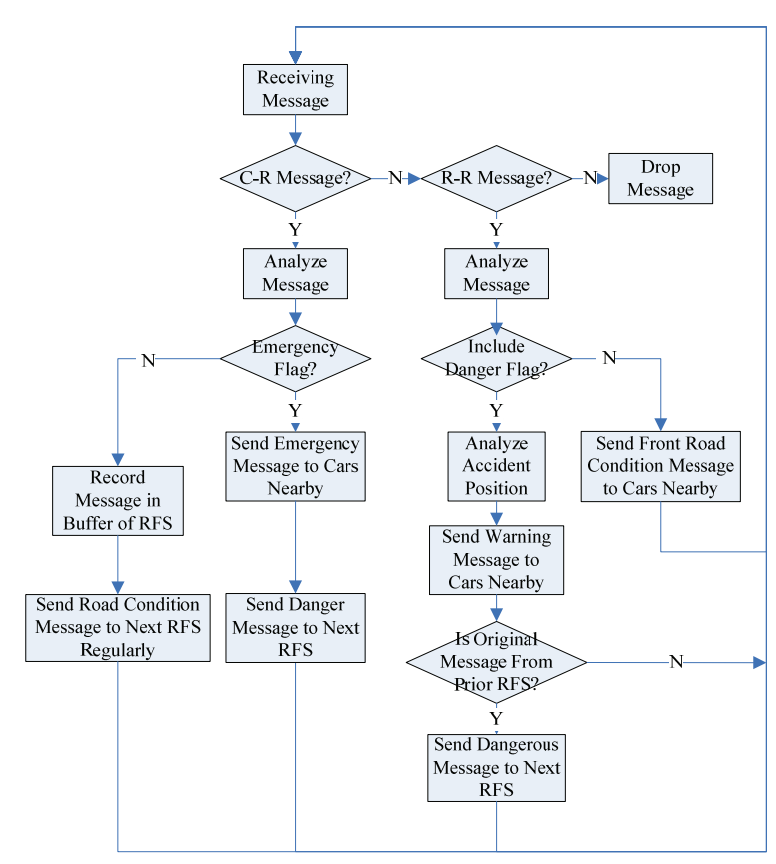

(b) Brief flowchart for the RFSs on the middle of the road. 


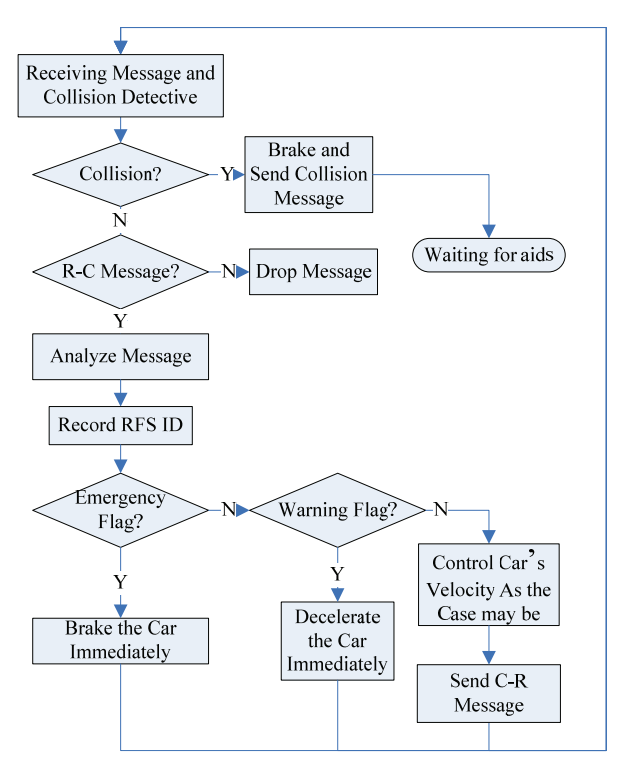

(c) Brief flowchart for the cars.

Figure 3. Brief flowcharts of "Cooperation" with RFSs.

Also, it is the same to cars, once cars cannot send their emergency messages or they cannot receive R-C messages from RFS, it will be very dangerous for either those failure cars or cars behind them.

Another criticality factor of this system is vehicles' number, we have to take into account that the bandwidth and the considered RFS's capability. While cars' number is very huge and thus bandwidth is not enough, and emergency messages may be drop in RFS's queue, which means it is blind of RFS if traffic accident happens around, and that case is not allowed absolutely.

And our solutions are listed as follows. First, we prepare spare RFSs and Car sensors in order to eliminate the error coursed by failures happen on sensors. We have to take the cost if the whole system into account, if we prepare too much sensors, it is a waste of money, so there is a dynamic balance between safeties and costs. Here we choose the scheme that each car has a spare sensor, and every four RFSs have a spare sensor. Second, we use priority queue to make sure emergency messages are not dropped by RFS while the condition of communication is in a dickey state. In [11], priority concept is brought forward firstly in vehicle ad-hoc network field. And in this system, we apply this comparatively mature idea, and we give some improvement to satisfy the need of safe navigation.

\section{Contrast Experiments}

In this section, we introduce our contrast experiments. Firstly we introduce the experimental platform, and then our contrast trial in highway scenarios will be shown in the second part, at the end of the second part, we give our analysis of this experiment.

\subsection{Experimental platform}

As is shown in Figure 4, we use HBEROBOCAR as our experimental platform. Figure 4(a) shown the car and Figure 4(b) shows the RFS. All the messages of this system are transported by ATmegal128L just as Figure 4(b) shows. Our car equips some sensors, such as PSD sensors and ultrasonic sensors so that it can detect forward obstacles, and also it quips one ATmegal128L interface so that it can communicate with RFSs while connecting with ATmegal128L node.

This car is $23 \mathrm{~cm}$ long and $15 \mathrm{~cm}$ wide, and $1: 18$ to the true car. The highest speed of the car is $47 \mathrm{~cm} / \mathrm{s}$. In this experiment, we pay our attention to the different speed after $60 \mathrm{~cm}$ of the traffic accident happens and warning distance between with and without RFSs.

\subsection{Experiments in Highway Scenarios}

We apply four cars and two RFSs to simulate the highway scenarios as is shown in Figure 5. Carl is the first car which would have an artificial accident thus we can measure car2 to car4's reaction time and stop distance.

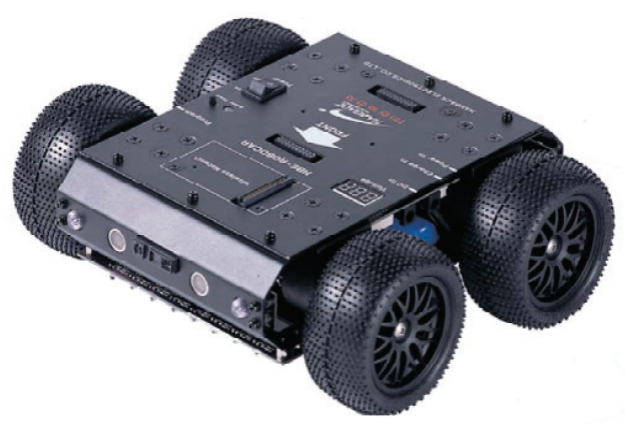

(a) One experimental car with PSD sensors and ultrasonic sensors.

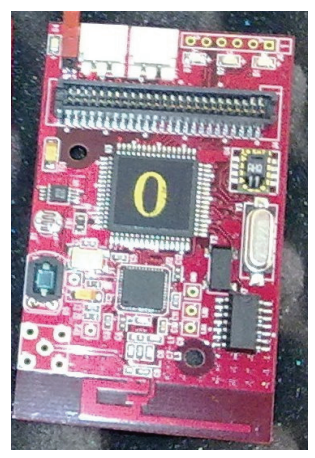

(b) RFS sensor used for communication

Figure 4. HBE-ROBOCAR experimental platform. 


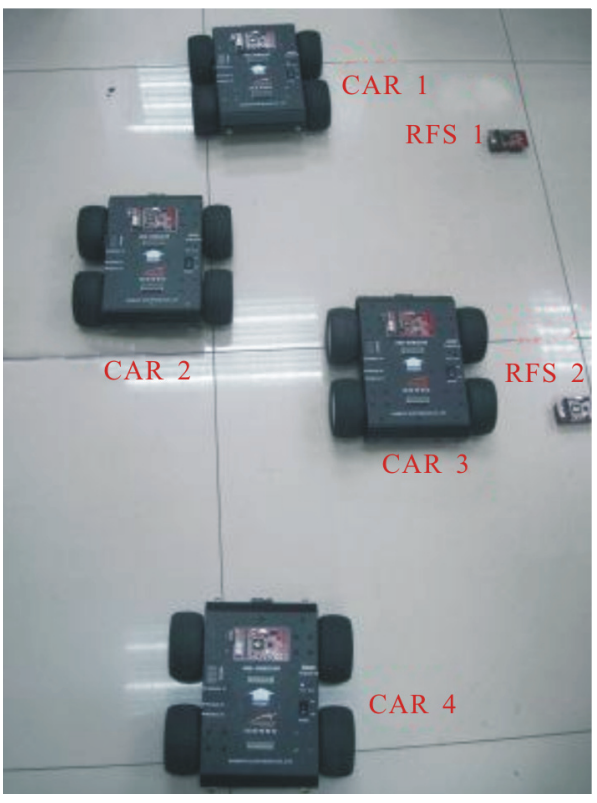

Figure 5. The simulative scenarios of our contrast experiments.

At First, we do not use RFSs and only four cars driving on the simulative scenarios. Suddenly the car runs upfront meet an accident, and we measure each car's speed when car runs into the $60 \mathrm{~cm}$ of the traffic accident and warning distance when accident happens. We will repeat for 20 times.

Then we add RFSs and with three RFSs and four cars, then we let the first car to have an accident just like the experiments above, then measure each car's speed and warning distance too. Also, we make the experiments for 20 times.

The results are shown in Figure 6 and consolidated measured data are shown in Table $\mathbf{1 .}$

As is shown in Figure 6 and Table 1 above, while adopting "cooperation" with RFS strategy, each car's speed at $60 \mathrm{~cm}$ after accident is much slower and the warning distance is much wider than the case without RFSs. Car2 is in front of Car3 and Car4, thus Car2's speed at $60 \mathrm{~cm}$ after accident is a little higher than Car3 and Car4. However, we can clearly find that the performance is improved obviously.

\section{Conclusions and Future Work}

In this paper, it is shown how to develop a novel safe navigation algorithm based on "cooperation" with RFSs, so as to improve the safety factor for the drivers while driving cars. Our experimental results show our algorithm effectively reduces the chain collision and extends the range of divers' situation awareness.

In the future, we focus our research priority on how to create a novel transport protocol so as to overcome

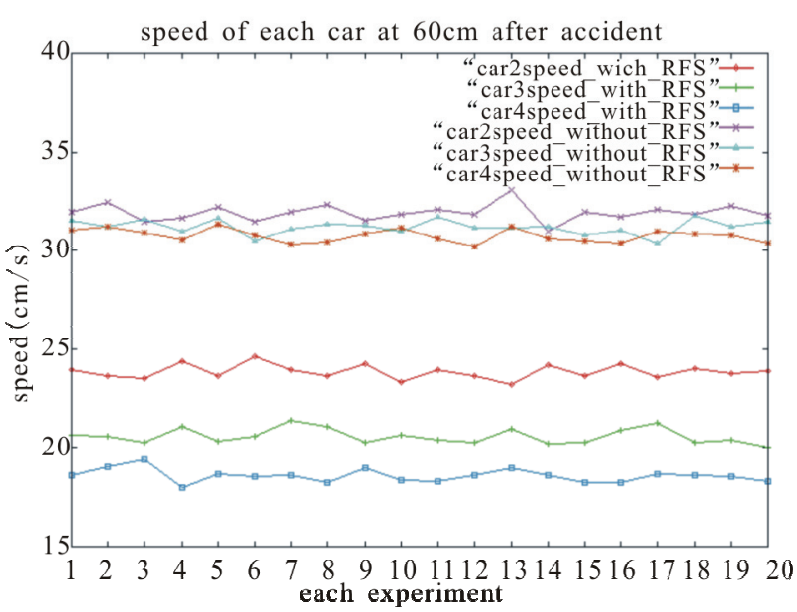

(a) Speed of each car at $60 \mathrm{~cm}$ after accident.

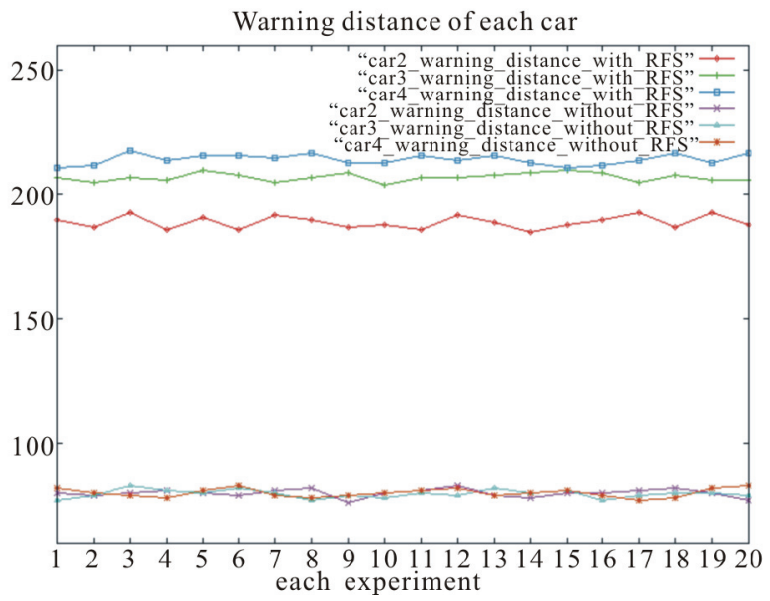

(b) Each car's warning distance

Figure 6. The result of contrast experiments of with and without RFSs.

Table 1. Average data of the contrast experiments.

\begin{tabular}{cccc}
\hline \multirow{2}{*}{ cars } & $\begin{array}{c}\text { Average data of measured } \\
\text { parameter }\end{array}$ & $\begin{array}{c}\text { without } \\
\text { RFSs }\end{array}$ & $\begin{array}{c}\text { with } \\
\text { RFSs }\end{array}$ \\
\hline \multirow{2}{*}{ car2 } & speed $(60 \mathrm{~cm})(\mathrm{cm} / \mathrm{s})$ & 31.93 & 23.86 \\
& warning distance $(\mathrm{cm})$ & 79.95 & 189.05 \\
\multirow{2}{*}{ car3 } & speed $(60 \mathrm{~cm})(\mathrm{cm} / \mathrm{s})$ & 31.22 & 20.59 \\
& warning distance $(\mathrm{cm})$ & 79.65 & 207.15 \\
\multirow{2}{*}{ car4 } & speed $(60 \mathrm{~cm})(\mathrm{cm} / \mathrm{s})$ & 30.77 & 18.58 \\
& warning distance $(\mathrm{cm})$ & 80.05 & 214.40 \\
\hline
\end{tabular}

wicked condition such as very high speed, too many cars and atrocious weather.

\section{Acknowledgements}

Supported by the Research Fund for the Doctoral Program of Higher Education of China(RFDP), the fund of the State Key Laboratory of Software Development En- 
vironment (Grant No. SKLSDE-2009ZX-04) and the National High-tech R\&D Program of China (863 Program) (Grant No. 2009AA043303, 2008AA12A216, 2009AA043305).

\section{References}

[1] G. Taylor, G. Blewitt, D. Steup, S. Corbett and A. Car, "Road Reduction Filtering for GPS-GIS Navigation," Transactions in GIS, Vol. 5, No. 3, 2001, pp. 193-207.

[2] R. Siegwart and I. R. Nourbakhsh. "Introduction to Autonomous Mobile Robots," MIT Press, Massachusetts, 2004.

[3] K. Macek, D. Vasquez, T. Fraichard and R. Siegwart. "Safe Vehicle Navigation in Dynamic Urban Scenarios," Proceeding of the 11th International IEEE Conference on Intelligent Transportation Systems, Beijing, October 2008, pp. 482-489.

[4] S. Petty and T. Fraichard. "Safe Motion Planning in Dynamic Environments," Proceedings of the IEEE International Conference on Intelligent Robots and Systems, Edmonton, 2005, pp. 2210-2215.

[5] P. Fiorini and Z. Shiller. "Motion Planning in Dynamic Environments Using Velocity Obstacles," International Journal of Robotics Research, Vol. 17, No. 7, July 1998, pp. 760-772.
[6] R. Simmons, "The Curvature Velocity Method for Local Obstacle Avoidance," International Conference on Robotics and Automation, Minneapolis, April 1996, pp. 3375-3382.

[7] H. Seraji, A. Howard and E. Tunstel. "Safe Navigation on Hazardous Terrain." Proceedings of the 2001 IEEE International Conference on Robotics \& Automation, Seoul, May 2001, pp. 3084-3091.

[8] S. Dietzel, B. Bako, E. Schoch and F. Kargl. "A Fuzzy Logic Based Approach for Structure-free Aggregation in Vehicular Ad-hoc Networks," Proceedings of the 6th ACM international workshop on Vehicular Internetworking, Beijing, September 2009, pp. 79-88.

[9] T. Fraichard and H. Asama, "Inevitable Collision States a Step towards Safer Robots?" Advanced Robotics, Vol. 18, No. 10, 2004, pp. 1001-1024.

[10] S. M. Jung, T. H. Song, J. H. Park, J. H. Park and J. W. Jeon, "The Safe Navigation of Remote Mobile Robot Using Virtual Stick," 2008 IEEE International Conference on Industrial Technology, Chengdu, April 2008, pp. 1295-1734.

[11] T. M. Marc, D. Jiang and H. Hartenstein. "Broadcast Reception Rates and Effects of Priority Access in 802.11-based Vehicular Ad-hoc Networks," Proceedings of the 1st ACM international workshop on Vehicular ad hoc networks, Philadelphia, October 2004, pp. 10-18. 